\title{
Observations of Energetic Storm Particles: An Overview
}

\author{
Christina M. S. Cohen \\ California Institute of Technology, Pasadena, California
}

\begin{abstract}
An increase in the intensity of energetic particles associated with the passage of an interplanetary shock has long been referred to as an 'energetic storm particle (ESP) event'. Such increases have been observed since the 1960's and the particles were generally thought to be either locally accelerated or trapped in the vicinity of the shock and transported with it. This general overview will describe the initial observations and theories of ESP events as well as the measurements that have led to our current understanding of the creation of these events. The relevance of ESP events to space weather as well as furthering our understanding of particle acceleration by shocks is also discussed.
\end{abstract}

\section{INTRODUCTION}

Energetic storm particle (ESP) events have been studied for over 30 years. This overview attempts to describe the general evolution of the observations and theories of ESP events from their first detection to our current understanding. Naturally, such an overview is not exhaustive and cannot present all the relevant measurements or past theories. However, hopefully, it provides a useful context and background for the current studies, relevance, and conventional wisdom of ESP events.

\subsection{Early Observations}

Increases in energetic particle intensities near the vicinity of a shock were first reported by Bryant et al. [1962]. Observations on 30 September 1961 from the cosmic ray instrument onboard Explorer 12 showed substantial increases in the 2-15 MeV proton intensities just after the sudden commencement of a geomagnetic storm. It was this association with a geomagnetic storm and the corresponding Forbush decrease that led Byant et al. to dub the ions 'energetic storm particles' (ESPs). The authors found the ESPs to have a softer spectrum than solar energetic particles (SEPs) and made the suggestion that they were solar protons trapped in a magnetic cloud region.

Several years later, Rao et al. [1967] reported on a survey of ESP events using cosmic ray detectors on the Pioneer 6 and 7 spacecraft. They found a one-to-one correspondence between the ESP events and Forbush decreases, as well as measurable anisotropies, which were not bi-directional, throughout the events. The authors argued against the ESPs being trapped in a magnetic cloud, primarily on the basis of the lack of bi-directional anisotropies, and suggested that interplanetary acceleration by the shock associated with the Forbush decrease was the cause of the enhanced intensities. 
Kahler [1969] disagreed after performing his own survey of ESP events associated with magnetic sudden commencements using data from Explorers 12, 33, 34, and 35, Mariner 4, OGOs 1 and 3, Pioneer 6, and IMPs 3 and 4. The author argued that the association of ESP events with SEP events suggested that both particle populations were generated at the Sun (n.b. this was during the time when SEP events were thought to originate solely from solar flares). Previously Lin et al. [1968] had suggested that SEP events were comprised of 3 components: the diffusive component which could populate a wide range of magnetic field lines as it moved through the interplanetary medium, followed by the core and halo components which were emitted over a narrow range of longitudes (the halo being made of higher energy particles and thus having a larger spatial extent than the core). Kahler argued that the latter two components were always accompanied by an interplanetary plasma cloud. Such a cloud could drag magnetic field lines outward, distorting them such that appropriately positioned spacecraft would intercept the field lines populated with halo protons and subsequently observe and increase in particle intensity, i.e., an ESP event.

\subsection{Early Theories}

By the early 1970's the dominant theories suggested ESP events were a result of 1) acceleration by bouncing between the Earth's bow shock and a traveling interplanetary shock [Axford and Reid, 1963], 2) diffusive shock acceleration at quasi-parallel interplanetary shocks [e.g., Jokipii, 1966; Scholer and Morfill 1975], 3) shock drift acceleration at quasi-perpendicular interplanetary shocks [e.g., Hudson, 1965], 4) sweeping by traveling magnetic structures [Palmer, 1972], or 5) trapping behind a moving interplanetary shock. Idea 1 was rejected when observations indicated that the distance between the bow shock and the position of the spacecraft during some ESP events was approximately an order of magnitude larger than the gyroradius of a typical ESP and not connected along nominal magnetic field lines indicating that particles were unlikely to be efficiently bouncing back and forth and gaining significant energy [Rao et al., 1967]. The theory of magnetic sweeping (item 4, above) was discounted when observations indicated that the increase in particle intensity often occurred behind the shock and not exclusively ahead of the shock as would be expected by the theory [Sarris and van Allen 1974]. Further, simulations were able to produce only ESP events significantly smaller than typically observed and even then required large diffusion coefficients and a totally reflecting shock, aspects not typical in interplanetary space [Palmer, 1972; Rao et al 1967]. Theory 5, trapping behind the shock, was not significantly pursued as an option as scientists focused heavily on shock-related acceleration (ideas 2 and 3, above). It was, however, preferred by Simnett and Holt 
[1970] as the explanation for an event observed by several spacecraft at different radial and longitudinal locations.

\section{CATEGORIZATION}

Sarris and van Allen [1974] proposed a two category system for ESP events based on the observed particle intensities: Spike events and classic ESP events. The characteristics are given in Table 1 . The primary observational difference was the size of the event and the theoretical distinction was believed to be the acceleration mechanism. In general, this categorization is still used today, however the more detailed measurements available from instruments such as the Electron Proton Alpha Monitor (EPAM) on the ACE spacecraft have lead to the defining of three additional categories to describe more complicated events [e.g., see Lario et al.2003 and Ho et al. 2005]. Figures 1 and 2 show examples of spike and classic ESP events (as identified by Ho et al. [2005]) using EPAM ion data.

\subsection{Spike Events}

Spike events are short in duration (5-10 minutes) and arrive within minutes of the shock. Observed increases in proton intensities rarely exceed $5 \mathrm{MeV}$. All of these characteristics are apparent in the event shown in Figure 1. It was proposed by Sarris and van Allen that these events are a result of shock drift acceleration at quasi perpendicular shocks.

The authors argued that the short duration of these events is chiefly a consequence of the difficulty in maintaining a perpendicular (or quasi-perpendicular) shock for extended periods of time. As effective acceleration is likely to occur only over a small angular regime (80-90 degrees), magnetic field fluctuations easily cause the shock orientation to move out of that range.

The limited time that a shock remains perpendicular also affects the typical maximum energy to which the particles are accelerated. High energy ions require longer times for acceleration and it was suggested that the orientation of a shock will typically be stable only long enough to produce particles with maximum energies around a few MeV.

These suggestions were supported by a series of spike events observed on 20 February 1968. Four distinct increases in the proton intensity were observed over the course of 3 hours. It was noticed that each event occurred when the orientation of the magnetic field was roughly 8090 degrees [see Figure 7 of Sarris and van Allen, 1974] with no increases observed when the field angle was smaller. Although only the first spike event was associated with a shock passage, the authors suggested that the subsequent events were 'remnants' of previous occasions when the shock was perpendicularly oriented. As these remnants diffused away from the shock they decreased in magnitude but remained large enough to be observed. 


\subsection{Classic ESP Events}

The durations of classic ESP (or simply ESP) events are typically longer than those of spike events according to Sarris and van Allen. ESP events can last for several hours and might arrive ahead or behind the shock. The maximum energy at which proton increases are observed is higher than for spike events, extending to $\sim 20 \mathrm{MeV}$. These characteristics can be seen in the event in Figure 2. In contrast to the spike event in Figure 1, the lowest energy ion intensities do not peak at the shock passage but slightly afterwards. The $>1 \mathrm{MeV}$ ion intensities for this classic event show a strong ESP increase while the spike event is barely evident at these energies in Figure 1. It should be noted that the maximum energy to which particles are being locally accelerated in an ESP event is still a topic of some discussion. Lario and Decker [2002] have presented evidence suggesting the large ESP event of 20 October 1989 (where increases were seen at energies $>500 \mathrm{MeV}$ ) was not the result of local shock acceleration (as presumed by Reames [1999b] and others) but was rather an earlier accelerated population trapped by a magnetic structure ahead of the shock and traveling with it. Regardless, the suggested source of the classic ESP events is diffusive shock acceleration at oblique or quasi-parallel shocks. As these events are generally bigger (in duration, energy extent, and intensity) there are more studies of classic ESP events, particularly in terms of composition and spectra.

The first study of heavy ion composition in such events was by Klecker et al. [1981] involving measurements of $\mathrm{He}, \mathrm{C}$, O, and $\mathrm{Fe}$. The composition data were obtained using the Ultra-Low-Energy-Wide-Angle-Telescope (ULEWAT) and the Ultra-Low-Energy-Charge-Analyzer (ULECA) on the ISEE-3 spacecraft. Additionally, charge state measurements were available from the Ultra-LowEnergy-Z-E-Q-Analyzer (ULEZEQ) on the same spacecraft. The authors found that during the ESP event on 28-29 September 1978 the heavy ion spectra steepened by an amount that depended on the particle's mass/charge (A/Q) ratio. This resulted in $\mathrm{Fe} / \mathrm{He}$ and $\mathrm{Fe} / \mathrm{O}$ abundance ratios that decreased during the event when measured at a constant energy/nucleon value. Klecker et al. suggested that the $\mathrm{A} / \mathrm{Q}$ effects were a result of diffusive shock acceleration involving rigidity-dependent mean-free path lengths.

More recent work on heavy ion composition in classic ESP events was reported by Desai et al. [2003]. This survey of 56 interplanetary shocks and the associated energetic particle increases revealed 25 cases of substantial amounts of ${ }^{3} \mathrm{He}$ being accelerated. The ${ }^{3} \mathrm{He} /{ }^{4} \mathrm{He}$ ratios varied between factors of 3 and 600 over the solar wind value. This, along with the overall heavy ion composition in these events, suggested that the solar wind was unlikely to be the sole seed population for these accelerated ions. The authors suggested the results could be best understood 
as the result of a $\mathrm{A} / \mathrm{Q}$-dependent acceleration of a seed population that itself was composed of multiple sources, including remnant flare material enriched in ${ }^{3} \mathrm{He}$.

\subsection{Anisotropy}

Measurements of the anisotropy of $35-1600 \mathrm{keV}$ proton increases during shock passages helped to seal the associations between spike events and shock drift acceleration and between ESP events and diffusive shock acceleration. Presenting two examples from a survey of 40 events, Wenzel et al. [1985] illustrated how the anisotropies downstream of a classic ESP event and a spike event differed substantially in the manner predicted by diffusive shock acceleration and shock drift acceleration respectively.

Proton data from the low-energy proton experiment on ISEE-3 revealed isotropic distributions for several hours during a large particle intensity increase associated with the passing of a shock on 5 April 1979. In contrast, data obtained during a short-duration spike coincident with a shock passage on 9 March 1979 showed pitch-angle distributions that were peaked at 90 degrees from the magnetic field direction immediately downstream of the shock. The plasma and magnetic field measurements from instruments on ISEE 3 indicated that the shock on 5 April 1979 was quasi-parallel with significant upstream wave activity, while the shock on 9 March 1979 was quasiperpendicular and had no wave activity upstream.

The particle, plasma, and field observations during the April event are consistent with the diffusive shock acceleration theory of Lee [1983]. His theory predicts upstream wave activity, downstream particle isotropy, and softening of the spectra as the shock approaches; all signatures seen in the ISEE 3 data from the April event but not in the March event. Analysis of acceleration by quasiperpendicular shocks by Decker [1983] resulted in particle anisotropies and intensity increases that matched those observed in the 9 March 1979 event indicating this event was likely the result of shock-drift acceleration.

In the larger survey of $\sim 40$ shock passages (examined regardless of the presence of an associated particle increase), it was found that the particle anisotropy downstream increased (as well as the occurrence of 90 degree peaked distributions) as the angle between the shock and the magnetic field, $\theta_{\mathrm{Bn}}$, increased [Sanderson et al., 1983]. It was thus concluded that quasi-perpendicular shocks $\left(\theta_{\mathrm{Bn}}>\sim 60^{\circ}\right)$ were a sign of shock-drift acceleration and quasi-parallel shocks $\left(\theta_{\mathrm{Bn}}<\sim 50^{\circ}\right)$ indicated diffusive shock acceleration. The authors observed more quasiperpendicular shocks than quasi-parallel ones, but found the larger particle increases were associated with quasiparallel shocks. This was consistent with the observations of van Nes et al. [1984] for shocks with $30^{\circ}<\theta_{\mathrm{Bn}}<60^{\circ}$ and suggested that diffusive shock acceleration was more 
efficient at accelerating $35-56 \mathrm{keV}$ protons than shock-drift acceleration.

\section{CURRENT UNDERSTANDING AND APPLICATION}

\subsection{Cause of ESP Events}

In 1995, Cane [1995] suggested that SEP and classic ESP events were basically the same things - enhanced particle intensities as the result of shock acceleration. As a shock moves from near the Sun toward $1 \mathrm{AU}$, it may accelerate particles along the way, some of which will escape upstream of the shock to be observed by spacecraft as an SEP event. If a spacecraft is located such that it intercepts a passing shock and the shock is strong enough to still be accelerating particles, an ESP event will be observed.

Using data from the Helios 1 and 2 and IMP-8 spacecraft, Cane was able to examine how the particle intensity time profiles in a given event differed when observed at different longitudes. Cases where the nose of the shock (the strongest part of the shock) passed over the spacecraft were most likely to involve an ESP event. It was also found that SEP events with an eastern origin (relative to the spacecraft) had typical time profiles: a gradual rise peaking at or after the passage of a shock (when one was observed). Western events also had a common profile that differed from eastern events: a fast rise in the particle intensities followed by an exponential decay. Thus, the observed evolution of the particle intensities (and the presence/absence of ESP events) depended on the properties of the shock (strength/speed), the spacecraft's magnetic connection to the shock, and how both of these aspects evolved as the shock moved outwards from the Sun.

This view was further supported by Kallenrode [1996] in a survey of 351 interplanetary shocks observed by the two Helios spacecraft and the associated increases in the $5 \mathrm{MeV}$ proton intensities. Kallenrode attempted to distinguish between particle intensity increases resulting from acceleration close to the Sun (and then propagating to the spacecraft) and those resulting from shock acceleration at or near the spacecraft. In cases where an ESP event was observed, this distinction was relatively straightforward. In other events where the increase was moderate, gradual, and not well peaked at the shock passage the distinction was more subjective. The local or interplanetary component of the events (i.e., typically the ESP event) was found to be larger, both absolutely and relative to the solar component, for observers closer to the flare normal, i.e., the nose of the shock. The size of the ESP event diminished when observed along the flanks of the shock.

The acceptance of a common acceleration mechanism for ESP and SEP events has sparked interest in ESP events as a potential way of understanding puzzling observations of SEP events. Contrary to expectations, initial results from Solar Isotope Spectrometer (SIS) on the ACE spacecraft 
found large SEP events with compositional signatures expected only in small, flare-related SEP events [Cohen et al., 1999]; specifically, large enhancements of heavy ions (e.g., $\mathrm{Fe} / \mathrm{O}$ ) above $10 \mathrm{MeV} /$ nucleon. Further measurements by instruments on ACE and Wind have confirmed these observations and there have been several attempts to find possible explanations [e.g., see Mason et al., 1999; Tylka et al., 2001; Mewaldt et al., 2003; Cohen et al., 2003; Cane et al., 2003; Tylka et al., 2005].

One suggested scenario involves the orientation of the shock as it accelerates particles near the Sun [Tylka et al., 2005]. This idea proposes that perpendicular shocks are more likely to produce enrichments of heavy ions than parallel shocks due to the higher injection energy required. This causes the accelerated ions to be drawn primarily from the suprathermal population, where presumably there is a larger contribution from flare SEP remnants, at perpendicular shocks. Since it is currently not possible to obtain the required measurements (near the Sun) to confirm or disprove this hypothesis, studying ESP events, where the shock orientation and particle composition can both be directly measured, may prove useful.

According to Tylka et al's idea, one might expect to see enhancements in heavy ions in spike events where shock acceleration at quasi-perpendicular shocks is occurring. On 23 May 2002 SIS observed roughly order of magnitude increases in heavy ion intensities (He-Fe) around 14 $\mathrm{MeV} /$ nucleon (Figure 3) in association with a shock passage at 10:15 UT. Fits to the data from the magnetometer (MAG) and solar wind plasma (SWEPAM) instruments on ACE indicate the shock $\theta_{\mathrm{Bn}}$ was $84^{\circ}$ (http://www-ssg.sr.unh.edu/mag/ace/ACElists/

obs_list.htm). Although abundance ratios for $\mathrm{N} / \mathrm{O}$ and $\mathrm{Mg} / \mathrm{O}$ did not change significantly, the $\mathrm{Fe} / \mathrm{O}$ ratio decreased by a factor of $\sim 5$ during the event (Figure 4 ) in contrast to expectations. However, it is hard to classify this event as a spike event in that the duration was atypically long ( $\sim 2$ hours) and it extended to unusually high energies. Additionally, other events with increased $\mathrm{Fe} / \mathrm{O}$ ratios at quasi-perpendicular shocks have been observed [e.g., see Figure 4 of Tylka et al. 2005] and low Fe/O ratios have been observed for shocks with a large range of $\theta_{\mathrm{Bn}}$ values (including quasi-perpendicular). Clearly more study is warranted.

\subsection{Space Weather}

With the continual presence of humans in space and more sensitive scientific equipment being stationed there, temporal changes in the radiation environment (especially near Earth) is currently a strong concern. While it has been established that proton intensities in SEP events are generally governed by streaming limits [Reames and $\mathrm{Ng}$, 1998], which can be theoretically calculated and predicted, these limits do not apply to ESP events. Large ESP events can result in dramatic, short-term increases in the particle 
intensities by more than an order of magnitude (particularly at lower energies, see Figure 5 or Figure 1 from Cohen et al. [2001]) creating a potentially unexpected, significant radiation hazard (see also, Reames [1999b]). Unfortunately, it is difficult to predict the size of an ESP event from the associated shock parameters. In fact, in a survey of 168 shock passages Lario et al. [2003] found that many shocks ( 65 or 113 out of 168 shocks for ions at 47-68 $\mathrm{keV}$ or $1.9-4.8 \mathrm{MeV}$, respectively) were not accompanied by an ESP event. Kallenrode [1996] also found that for a given shock speed, the corresponding particle increase at 5 $\mathrm{MeV}$ varied by up to 6 orders of magnitude. Similarly, van Nes [1984] found a $\sim 2-3$ order of magnitude spread in 35 $\mathrm{keV}$ proton intensities for a given shock strength.

Even the spectral characteristics of ESP events are not easily predicted from shock parameters. Although diffusive shock acceleration models, such as that of Lee [1983], suggest that the spectral index of the accelerated ion population should be a simple function of the shock compression ratio, this is not seen in the observations. Desai et al. [2004] found the spectral index of oxygen in ESP events was not well correlated with the measured shock compression ratio. Thus predicting the size of an ESP event and the maximum energy to which it will be seen is unreliable when based on measured shock properties. Further, the shock properties themselves are not currently well predicted.

A less desirable, but more obtainable, alternative to forecasting ESP events is nowcasting. This involves reporting in situ measurements of shocks and ESP events in real time from spacecraft far enough upstream of the Earth to provide some degree of warning. The ACE spacecraft is currently orbiting the L1 Lagrangian point and can provide 30-60 minutes of warning for near-Earth operations (e.g., space shuttle flights, Earth orbiting satellites). This is illustrated in Figure 5 where an ESP event is seen first by SIS on ACE and then subsequently by the energetic particle detector on the GOES-8 spacecraft orbiting Earth. The similarity of the intensity time profiles is remarkable and leaves little doubt that the two spacecraft were observing the same shock, which was probably still accelerating particles. Comparison of the time profiles indicates ACE observed the shock and related ESP event $\sim 45$ minutes before GOES. Other examples are presented and discussed in Cohen et al. [2001].

Work is currently in progress to develop an automated shock identification routine using the real-time magnetic field and solar wind data from the MAG and SWEPAM instruments, respectively, on ACE. These data will be combined with real-time proton data from 50 to $5000 \mathrm{keV}$, and at 10 and $30 \mathrm{MeV}$ from the EPAM and SIS instruments, respectively, in order to assess the presence and strength of ESP events at ACE. The ACE real-time data are typically available within 5 minutes of acquisition, thus a quick automated evaluation of ESP events could provide warnings of incoming particle-accelerating shocks 
30-60 minutes before they reach sensitive equipment/personnel. This could be enough time to place sensors into protected configurations, cancel or end extravehicular activities of astronauts, and re-evaluate launch conditions for rockets/shuttles.

The real-time EPAM proton intensities are also currently being used to predict shock arrival times [Vandegriff et al. 2005]. As these ions escape from the shock region, they are detected upstream and form a 'ramp' towards the shock passage, which is evaluated by a neural network. The results provide a 24 hour advance prediction of the shock arrival time with an uncertainty of 8.9 hours, which is reduced to 4.6 hours for a 12 hour advance prediction. Similar work done by Posner et al. [2004], using Wind/STICS suprathermal ion measurements, indicated 572 hours of advanced warning was possible. Although these techniques do not evaluate the size of the ESP event or its potential hazard they do provide longer advanced warning of incoming strong shocks than nowcasting efforts.

\section{SUMMARY}

Over the course of $>30$ years the causes of shockassociated particle increases, called energetic storm particle events, have been debated and data examined until the scientific community formed a general consensus. The larger, classical ESP events are a result of diffusive shock acceleration occurring as a shock passes the spacecraft; generally the same process that creates most large SEP events. The locally-accelerated particle population is usually isotropic and reflects a rigidity-dependent process acting on the upstream suprathermal seed population. Spike events are the short-lived results of shock drift acceleration at quasi-perpendicular shocks. These events exhibit strong anisotropies and are typically a low-energy phenomena.

The importance of understanding the causes and characteristics of ESP events is both fundamental and practical. Studying ESP events is currently the most useful way to examine interplanetary shock acceleration in general as the parameters of the shock can be measured directly, something currently not possible for SEP acceleration close to the Sun (where most $>10$ $\mathrm{MeV} /$ nucleon ions are accelerated). In a practical sense, ESP events can be a direct radiation hazard for space-based assets. Understanding their creation is key to predicting them, something not presently possible but highly desirable.

The statement by Gombosi et al. [1979] is unfortunately still applicable over 25 years later: 'Further progress in deeper understanding on interplanetary shock associated energetic particle events may heavily depend on new simultaneous, spatially separated spacecraft observations with comprehensive, high-resolution instrumentation and 
data analysis.' Hopefully, new results from the STEREO mission in 2006 will fit the bill.

Acknowledgments. This work was supported by NASA at the California Institute of Technology (under grant NAG5-6912). I thank the EPAM, MAG, and SWEPAM teams and the ACE Science Center for making their data available online. GOES data were obtained through the SEC/NOAA website. I thank R.A. Leske for carefully reading this manuscript and his helpful comments, T.T. von Rosenvinge for providing Figures 3 and 4, and the organizers for putting together a stimulating and enjoyable conference.

\section{REFERENCES}

Axford, W.I., and G.C. Reid, Increases in Intensity of Solar Cosmic Rays before Sudden Commencements of Geomagnetic Storms, Journal of Geophysical Research, 68, 1793-1803, 1963.

Bryant, D.A., T.L. Cline, U.D. Desai, and F.B. McDonald, Explorer 12 Observations of Solar Cosmic Rays and Energetic Storm Particles after the Solar Flare of September 28, 1961, Journal of Geophysical Research, 67, 4983-5000, 1962.

Cane, H.V., The Structure and Evolution of Interplanetary Shocks and the Relevance for Particle Acceleration, Nuclear Physics B - Proceedings Supplements, 39 (1), 35-44, 1995.

Cane, H.V., T.T. von Rosenvinge, C.M.S. Cohen, and R.A. Mewaldt, Two Components in Major Solar Particle Events, Geophysical Research Letters, 30, SEP 5-1, doi: 10.1029/2002GL016580, 2003.

Cohen, C.M.S., R.A. Mewaldt, A.C. Cummings, R.A. Leske, E.C. Stone, P.L. Slocum, M.E. Wiedenbeck, E.R. Christian, and T.T. von Rosenvinge, Forecasting the Arrival of Shock-Accelerated Solar Energetic Particles at Earth, Journal of Geophysical Research, 106, 20979-20984, 2001.

Cohen, C.M.S., R.A. Mewaldt, A.C. Cummings, R.A. Leske, E.C. Stone, T.T. von Rosenvinge, and M.E. Wiedenbeck, Variability of Spectra in Large Solar Energetic Particle Events, Advances in Space Research, 32, 2649-2654, 2003.

Cohen, C.M.S., R.A. Mewaldt, R.A. Leske, A.C. Cummings, E.C. Stone, M.E. Wiedenbeck, E.R. Christian, and T.T. von Rosenvinge, New Observations of Heavy-Ion-Rich Solar Particle Events from ACE, Geophysical Research Letters, 26, 2697-2700, 1999.

Decker, R.B., Formation of Shock-Spike Events at QuasiPerpendicular Shocks, Journal of Geophysical Research, 88, 9959-9973, 1983.

Desai, M.I., G.M. Mason, J.R. Dwyer, J.E. Mazur, C.W. Smith, and R.M. Skoug, Evidence for a Suprathermal Seed Population of Heavy Ions Accelerated by Interplanetary Shocks near $1 \mathrm{AU}$, Astrophysical Journal, 588, 1149-1162, 2003.

Desai, M.I., G.M. Mason, M.E. Wiedenbeck, C.M.S. Cohen, J.E. Mazur, J.R. Dwyer, R.E. Gold, S.M. Krimigis, Q. Hu, C.W. Smith, and R.M. Skoug, Spectral Properties of Heavy Ions Associated with the Passage of Interplanetary Shocks at 1 AU, Astrophysical Journal, 611, 1156-1174, 2004.

Gombosi, T., K. Kecskemety, and S. Pinter, On the Connection of Interplanetary Shock Wave Parameters and Energetic Storm Particle Events, Geophysical Research Letters, 6, 313-316, 1979.

Ho, G.C., D. Lario, R.B. Decker, M.I. Desai, Q. Hu, C.W. Smith, and R.M. Skoug, Transient Shocks and Associated Energetic 
Particle Distributions Observed by ACE during Solar Cycle 23, Journal of Geophysical Research, submitted.

Hudson, P.D., Reflection of Charged Particles by Plasma Shocks, Monthly Notices of the Royal Astronomical Society, 131, 23-50, 1965.

Jokipii, J.R., Pitch-Angle Dependence of First-Order Fermi Acceleration at Shock Fronts, Astrophysical Journal, 145, 616622, 1966

Kahler, S.W., A Comparison of Energetic Storm Protons to Halo Protons, Solar Physics, 8, 166-185, 1969.

Kallenrode, M.-B., A Statistical Survey of 5-MeV Proton Events at Transient Interplanetary Shocks, Journal of Geophysical Research, 101, 24393-24410, 1996.

Klecker, B., M. Scholer, D. Hovestadt, G. Gloeckler, and F.M. Ipavich, Spectral and Compositional Variations of Low Energy Ions During an Energetic Storm Particle Event, Astrophysical Journal, 251, 393-401, 1981.

Lario, D. and R.B. Decker, The Energetic Storm Particle Event of October 20, 1989, Geophysical Research Letters, 29, 1393, 10.1029/2001GL014017, 2002.

Lario, D., G.C. Ho, R.B. Decker, E.C. Roelof, M.I. Desai, and C.W. Smith, ACE Observations of Energetic Particles Associated with Transient Interplanetary Shocks, in AIP Conf. Proc. 679: Solar Wind Ten, pp. 640-643, 2003.

Lee, M.A., Coupled Hydromagnetic Wave Excitation and Ion Acceleration at Interplanetary Traveling Shocks, Journal of Geophysical Research, 88, 6109-6119, 1983.

Lin, R.P., S.W. Kahler, and E.C. Roelof, Solar Flare Injection and Propagation of Low-Energy Protons and Electrons in the Event of 7-9 July, 1966, Solar Physics, 4, 338-360, 1968.

Mason, G.M., C.M.S. Cohen, A.C. Cummings, J.R. Dwyer, R.E. Gold, S.M. Krimigis, R.A. Leske, J.E. Mazur, R.A. Mewaldt, E. Mobius, M. Popecki, E.C. Stone, T.T. von Rosenvinge, and M.E. Wiedenbeck, Particle Acceleration and Sources in the November 1997 Solar Energetic Particle Events, Geophysical Research Letters, 26, 141-144, 1999.

Mewaldt, R.A., C.M.S. Cohen, G.M. Mason, M.I. Desai, R.A. Leske, J.E. Mazur, E.C. Stone, T.T. von Rosenvinge, and M.E. Wiedenbeck, Impulsive Flare Material: A Seed Population for Large Solar Particle Events?, in 28th International Cosmic Ray Conference, pp. 3229-3232, Tsukuba, Japan, 2003.

Palmer, I.D., Shock Wave Effects in Solar Cosmic Ray Events, Solar Physics, 27, 466-477, 1972.

Posner, A., N.A. Schwadron, D.J. McComas, E.C. Roelof, and A.B. Galvin, Suprathermal ions ahead of Interplanetary Shocks: New Observations and Critical Instrumentation Required for Future Space Weather Monitoring, Space Weather Journal, 2, S100004, 10.1029/2004SW000079, 2004.

Reames, D.V., Particle Acceleration at the Sun and in the Heliosphere, Space Science Review, 90, 413-491, 1999a.

Reames, D.V., Solar Energetic Particles: Is There Time to Hide?, Radiation Measurements, 30, 297-308, 1999b.

Reames, D.V. and C.K. Ng, Streaming-limited Intensities of Solar Energetic Particles, Astrophysical Journal, 504, 1002-1005, 1998.

Rao, U.R., K.G. McCracken, and R.P. Bukata, Cosmic-Ray Propagation Processes, Journal of Geophysical Research, 72 (17), 4325-4341, 1967.

Sanderson, T.R., R. Reinhard, and K.-P. Wenzel, Anisotropies of 35-56 keV Ions Associated with Interplanetary Shocks, in 18th International Cosmic Ray Conference, pp. 156-159, Bangalore, India, 1983. 
Sarris, E.T., and J.A. van Allen, Effects of Interplanetary Shock Waves on Energetic Charged Particles, Journal of Geophysical Research, 79, 4157-4173, 1974.

Scholer, M. and G.E. Morfill, Simulation of Solar Flare Particle Interaction with Interplanetary Shock Waves, Solar Physics, 45, 227-240, 1975.

Simnett, G.M., and S.S. Holt, Long Term Storage of Relativistic Particles in the Solar Corona, Solar Physics, 16, 208-223, 1970.

Tylka, A.J., C.M.S. Cohen, W.F. Dietrich, M.A. Lee, C.G. Maclennan, R.A. Mewaldt, C.K. Ng, and D.V. Reames, Shock Geometry, Seed Populations, and the Origin of Variable Elemental Composition at High Energies in Large Gradual Solar Particle Events, Astrophysical Journal, 625, 474-495, 2005.

Tylka, A.J., C.M.S. Cohen, W.F. Dietrich, C.G. Maclennan, R.E. McGuire, C.K. Ng, and D.V. Reames, Evidence for Remnant Flare Suprathermals in the Source Population of Solar Energetic Particles in the 2000 Bastille Day Event, Astrophysical Journal, 558, L59-L63, 2001.

van Nes, P., R. Reinhard, T.R. Sanderson, K.-P. Wenzel, and R.D. Zwickl, The Energy Spectrum of 35- to $1600-\mathrm{keV}$ Protons Associated with Interplanetary Shocks, Journal of Geophysical Research, 89, 2122-2132, 1984.

Vandegriff, J. K., K. Wagstaff, G. Ho, and J. Plauger, Forcasting space weather: predicting energetic store particle events using neural networks, Advances in Space Research, in press, 2005.

Wenzel, K.-P., R. Reinhard, T.R. Sanderson, and E.T. Sarris, Characteristics of Energetic Particle Events Associated with Interplanetary Shocks, Journal of Geophysical Research, 90, 12-18, 1985.

Christina M. S. Cohen, California Institute of Technology, MC 220-47, Pasadena, CA 91125 USA

\section{FIGURE CAPTIONS}

Figure 1. Example of a spike ESP event (2002 day 149), showing ion intensities from the EPAM LEMS120 telescope as a function of time from the shock passage (at 15:04 UT as identified by the MAG and SWEPAM instruments). Energy ranges for each trace are given to the right of the figure.

Figure 2. Example of a classic ESP event (2002 day 238), showing ion intensities from the EPAM LEMS120 telescope as a function of time from the shock passage (at 06:21 UT as identified by the MAG and SWEPAM instruments). Energy ranges for each trace are given to the right of the figure.

Figure 3. Heavy ion intensities (at $14 \mathrm{MeV}$ /nucleon) from the SIS instrument as a function of time showing an ESP event. The time of shock passage is indicated by the ' $\mathrm{S}$ ' and line at the center of the plot (courtesy of T.T. von Rosenvinge).

Figure 4. Elemental ratios at $14 \mathrm{MeV} /$ nucleon as a function of time derived from the intensities shown in the previous figure, normalized by values of Reames [1999a]. The time of shock passage is indicated by the ' $\mathrm{S}$ ' and line at the center of the plot (courtesy of T.T. von Rosenvinge)

Figure 5. Integral intensities of $>10 \mathrm{MeV}$ protons from SIS/ACE and GOES- 8 for an energetic storm particle event. 
Figure 1. Example of a spike ESP event (2002 day 149), showing ion intensities from the EPAM LEMS120 telescope as a function of time from the shock passage (at 15:04 UT as identified by the MAG and SWEPAM instruments). Energy ranges for each trace are given to the right of the figure.

Figure 2. Example of a classic ESP event (2002 day 238), showing ion intensities from the EPAM LEMS120 telescope as a function of time from the shock passage (at 06:21 UT as identified by the MAG and SWEPAM instruments). Energy ranges for each trace are given to the right of the figure.

Figure 3. Heavy ion intensities (at $14 \mathrm{MeV}$ /nucleon) from the SIS instrument as a function of time showing an ESP event. The time of shock passage is indicated by the ' $\mathrm{S}$ ' and line at the center of the plot (courtesy of T.T. von Rosenvinge).

Figure 4. Elemental ratios at $14 \mathrm{MeV} /$ nucleon as a function of time derived from the intensities shown in the previous figure, normalized by values of Reames [1999a]. The time of shock passage is indicated by the ' $S$ ' and line at the center of the plot (courtesy of T.T. von Rosenvinge).

Figure 5. Integral intensities of $>10 \mathrm{MeV}$ protons from SIS/ACE and GOES- 8 for an energetic storm particle event.

ESP MEASUREMENTS OVERVIEW

CHRISTINA M. S. COHEN

Table 1: Proton Characteristics of Spike and Classic ESP Events (adapted from Sarris and van Allen [1974])

\begin{tabular}{|l|l|l|}
\hline Characteristic & Spike Event & Classic Event \\
\hline Duration & $5-20$ minutes & Several hours \\
Arrival & $\begin{array}{l}\text { Within 5-10 } \\
\text { minutes of shock }\end{array}$ & $\begin{array}{l}\text { Ahead or } \\
\text { behind shock }\end{array}$ \\
$\begin{array}{l}\text { Maximum } \\
\begin{array}{l}\text { Energy } \\
\text { Acceleration } \\
\text { Mechanism }\end{array}\end{array}$ & $\sim 5 \mathrm{MeV}$ & $\sim 20 \mathrm{MeV}$ \\
$\begin{array}{l}\text { Shock } \\
\text { orientation }\end{array}$ & $\begin{array}{l}\text { Shock Drift } \\
\text { perpendicular }\end{array}$ & Diffusive Shock \\
\hline
\end{tabular}




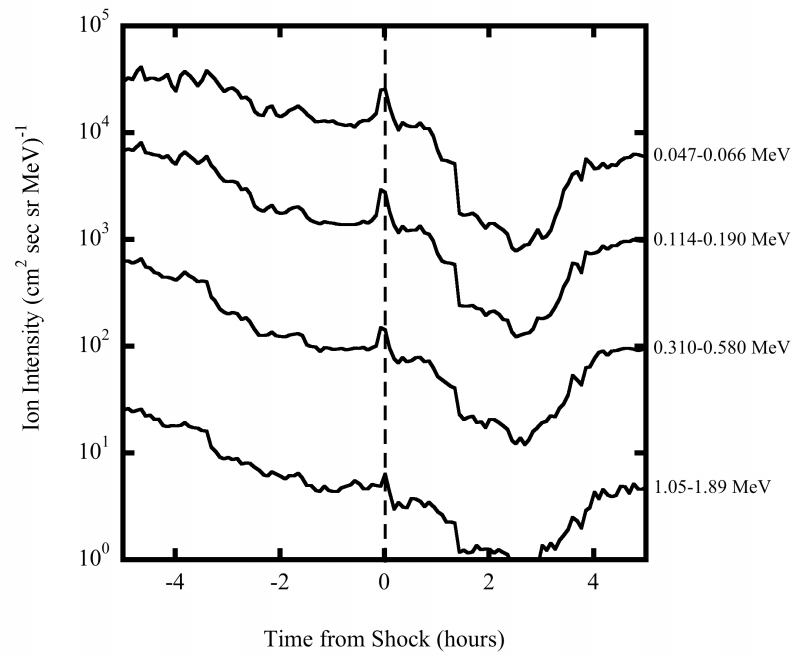

Figure 1.

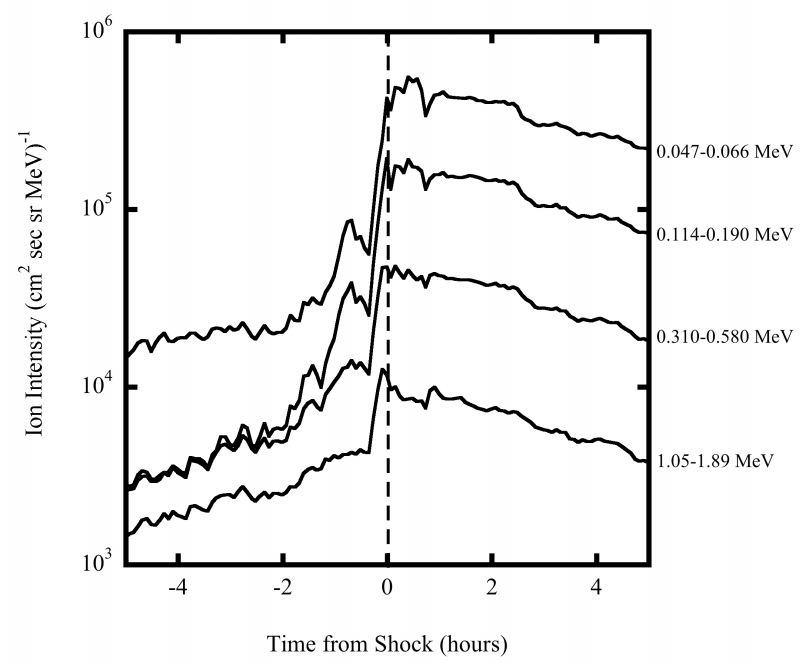

Figure 2. 


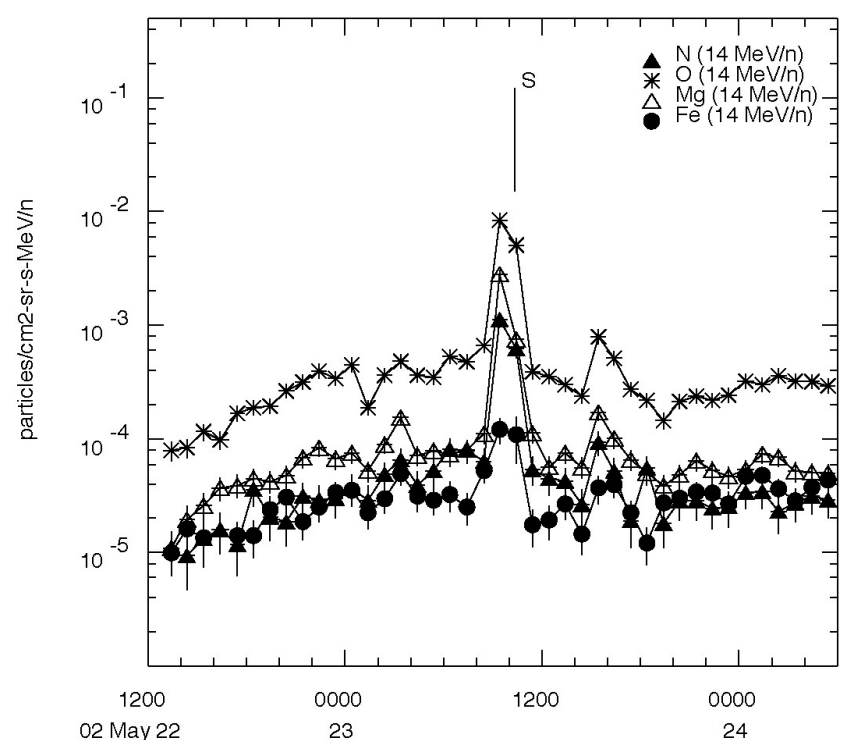

Figure 3.

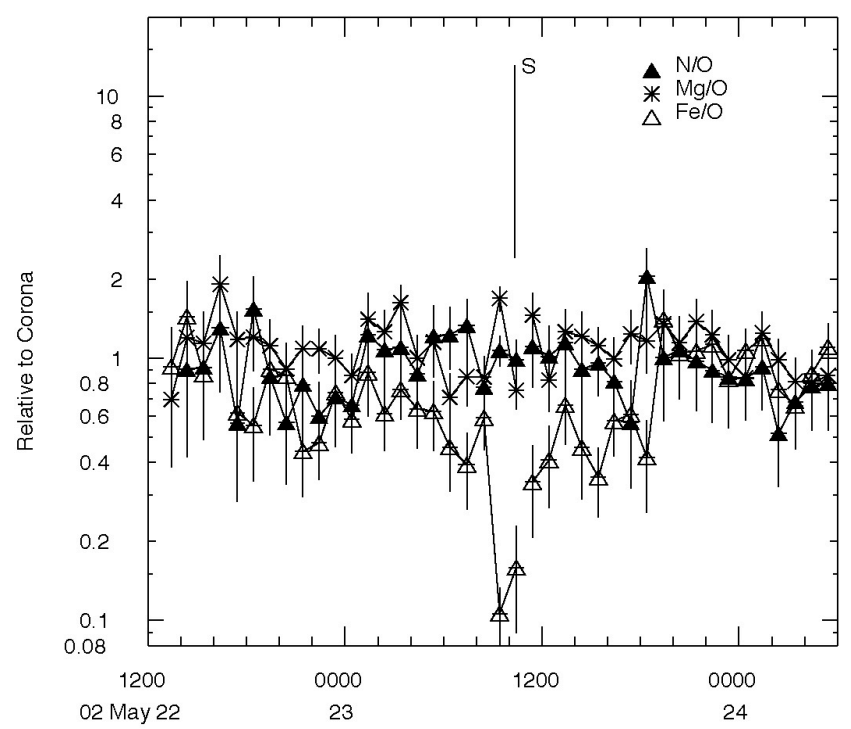

Figure 4. 


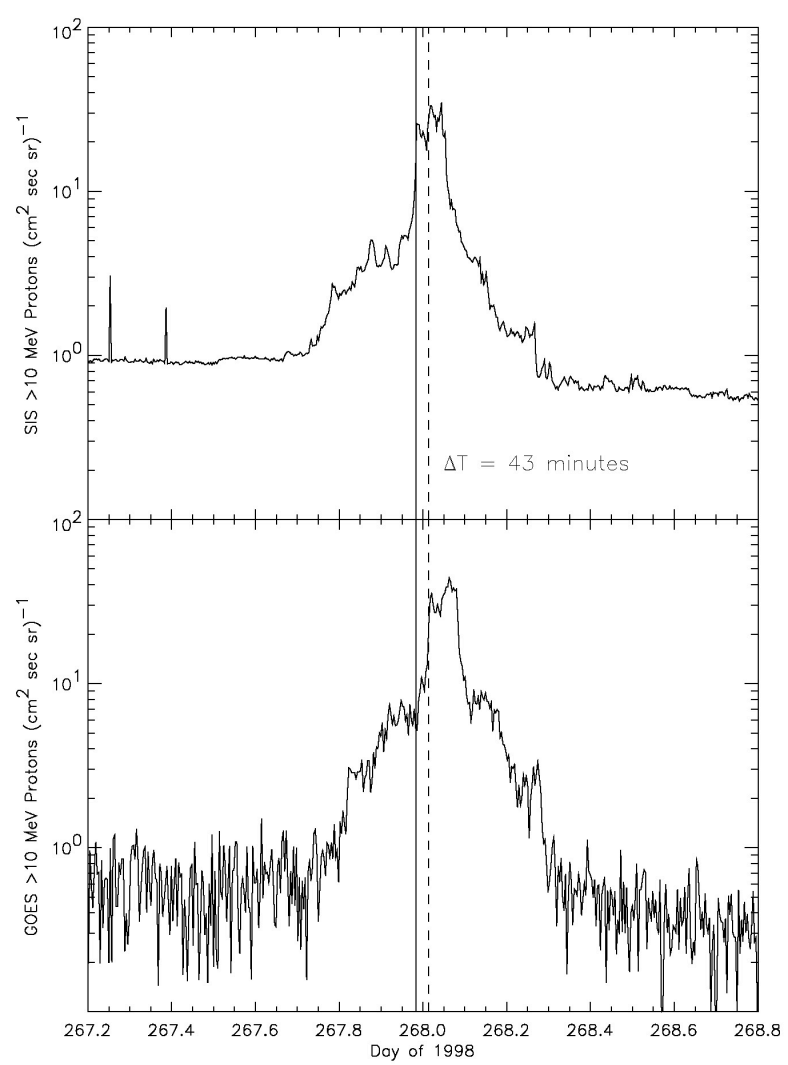

Figure 5. 